\title{
Performance Comparison of Different Affine Projection Algorithms for Noise Minimization from Speech Signals
}

\author{
Deepak Gupta $^{1}$, V K Gupta ${ }^{2}$, Mahesh Chandra ${ }^{3}$ \\ ${ }^{1}$ Krishna Engineering College, Ghaziabad, India \\ ${ }^{2}$ Inderprastha Engineering College, Ghaziabad, India \\ ${ }^{3}$ Birla Institute of Technology, Mesra, Ranchi, India \\ 1979.deepak@gmail.com, ${ }^{2}$ guptavk76@gmail.com, ${ }^{3}$ shrotriya@bitmesra.ac.in
}

\begin{abstract}
In this paper, different variants of affine projection algorithms (APAs) are implemented for noise minimization from speech signals. The variants of APA implemented are R-APA, VSS-RAPA and R-APSA. The performances of these algorithms are compared for various parameters like SNR improvement, mean square error (MSE), convergence rate, robustness and computational complexity. Results are computed on mathematical simulation of noise cancellation system on Matlab. Noisy speech samples corrupted by F16, babble16, car and factory noises at OdB, -5dB, -10dB and -20dB SNR levels for both single word and sentence are used for simulation at different filter orders. It is observed that the R-APSA algorithm outperforms among all algorithms at all filter orders and all input SNR levels. The R-APSA algorithm shows a maximum of $21.2 \mathrm{~dB}$ of improvement in SNR at -20dB input SNR level at filter order 5.
\end{abstract}

Keywords: Adaptive Filter, Adaptive Noise Cancellation, Signal to Noise Ratio (SNR), MSE, R-APA, VSS-RAPA, R-APSA

\section{Introduction}

In the current era, with the evolution of voice operated devices, the problem of noise merging with the audio is a major area of research especially for the applications of speech operated devices where noise minimization is essential. Noise gets automatically embedded into the signal which reduces the quality and intelligibility of the speech signals. Noise can be reduced by the use of proposed approach known as Active Noise Cancellation (ANC) [1-2]. The adaptive filters are mostly preferred for cancellation of noise from speech signals in the areas such as acoustic, speech, communication etc.

Adaptive filter algorithms are of two types, Minimum Mean Square Error criteria based and Recursive Least Squares criteria based algorithm [3-5]. The recently developed adaptive algorithms are evolved from these two basic algorithms. The low computational complexity is the unique feature of Least Mean square (LMS) algorithm. Though variation of step size improves the convergence rate of conventional LMS algorithm but faster convergence is required for improved performance.

On the other hand, Recursive Least Square (RLS) overcomes disadvantages of LMS as it has fast convergence. However computational complexity is increased in RLS algorithm. Affine projection algorithms (APAs) are proposed to provide optimum performance in terms of computational complexity and convergence rate. In this work, the Regularized Affine Projection Algorithm (R-APA) is implemented. Further its performance is improved by taking variable step size instead of constant value. The resulting algorithm is called as Variable Step Size Regularized Affine Projection Algorithm (VSS-RAPA). Since R-APA and VSS-APA suffers from high computational 
complexity because of matrix inversion, Regularized Affine Projection Sign Algorithm (R-APSA) is used for noise cancellation. The general setup of an adaptive noise canceller [6] is shown in the Fig1.

The input to the FIR filter is the reference noise $\tilde{N}(k)$ is. The output of the FIR filter which is the convoluted version of reference noise and the filter coefficients is $y(k)$. The $s(k)$ is the information signal which are corrupted with noise $N(k)$ making noisy signal $d(k)$. By subtracting the filter output $y(k)$ from noisy signal $d(k)$ the error signal $e(k)$ is obtained. The filter coefficients are changed in each iteration. This results when the reference noise approaches the amount of noise present in noisy signal, error signal equals the noiseless version of the information signal.

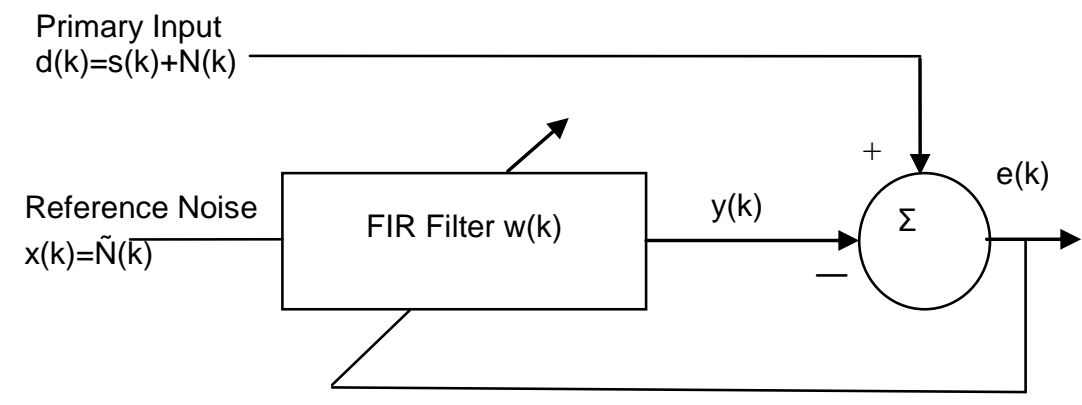

Figure 1. An Adaptive Noise Cancellation System

\section{APA Algorithms}

An affine projection algorithm (APA) and its variants have been proposed in recent years [7-9]. The weight updation of APA algorithm depends on multiple recent past input vectors where as in LMS and NLMS algorithms the past input vectors are not considered. In APA algorithms as the projection order increases the convergence rate increases but this result also increase in computational complexity.

\subsection{R-APA Algorithm}

The R-APA variables used in adaptive noise cancellation system are the excitation noise signal matrix for adaptive filter, $X(k)$, which is L by M matrix, the desired signal is the corrupted speech signal $\mathrm{d}(\mathrm{k})$. Here projection order of APA is $\mathrm{M}$ and length of filter is $\mathrm{L}$. The weight update equation for classical regularized APA is stated as

$$
w(k)=w(k-1)+\mu X(k)\left[X^{T}(k) X(k)+\delta I\right]^{-1} e(k)
$$

In equation (1), $\mu$ is the step size $(0 \leq \mu \leq 1)$, error vector is $e(k)=d(k)-y(k)$ and filter output is $y(k)=X^{T}(k) w(k-1), \delta$ is called as the regularization parameter.

\subsection{VSS-RAPA Algorithm}

The VSS-RAPA algorithm gives improved performance than R-APA. This is due to the variable step size in VSS-RAPA than constant step size in RAPA [10-12]. The summary of the VSS-RAPA algorithm [12] is as follows -

Initialization: $\hat{w}(0)=0_{L^{*} 1}, \hat{\sigma}_{d}^{2}(0)=0, \hat{\sigma}_{\hat{y}}^{2}(0)=0$

For $l=0$ to $M-1$

$$
\sigma_{e l+1}^{2}(0)=0
$$


For time index $k=1,2, \ldots$

$$
\begin{gathered}
\hat{e}(k)=d(k)-X^{T}(k) w(k-1) \\
y(k)=X^{T}(k) w(k-1) \\
\sigma_{d}^{2}(k)=\lambda \sigma_{d}^{2}(k-1)+(1-\lambda) d^{2}(k) \\
\sigma_{y}^{2}(k)=\lambda \sigma_{y}^{2}(k-1)+(1-\lambda) y^{2}(k)
\end{gathered}
$$

For $l=0$ to $M-1$

$$
\begin{array}{r}
\sigma_{e l+1}^{2}(k)=\lambda \sigma_{e l+1}^{2}(k-1)+(1-\lambda) e_{l+1}^{2}(k) \\
\mu_{l}(k)=\left|1-\frac{\sqrt{\left|\hat{\sigma}_{d}^{2}(k-1)-\hat{\sigma}_{\hat{y}}^{2}(k-1)\right|}}{\xi+\hat{\sigma}_{e(l+1)}^{2}(k)}\right| \\
\mu(k)=\operatorname{diag}\left\{\mu_{0}(k), \mu_{1}(k), \ldots ., \mu_{M-1}(k)\right\} \\
w(k)=w(k-1)+X(k)\left[\delta I_{p}+X^{T}(k) X(k)\right]^{-1} \mu(k)
\end{array}
$$

In equation (2), the value of the regularization parameter is decided based upon the noise level which corrupts the signal. For low SNR the value of regularization parameter required is high. The value of projection order of algorithm is also a deciding factor for regularization factor.

\subsection{VSS-RAPA Algorithm}

At high cost the APA algorithm converges fast in terms of computational complexity but at the same time in the because of impulsive noises it suffers performance degradation. Family of sign algorithms are preferred by the researchers because of its low complexity. Also it is more robust for impulsive noise than other algorithms. The Regularized Affine Projection Sign Algorithm (R-APSA) [13-14] adds the advantages of the R-APA and sign algorithms. In R-APSA L1-norm optimization criterion is used for weight updation when multiple projections are taken. By the use of APSA the convergence rate at a low computational complexity is improved.

An improved R-APA algorithm can be obtained by taking a posteriori error vector as follows [13]-

$$
\begin{gathered}
\min _{\hat{W}(k+1)}\left\|y(k)-X^{T}(k) \hat{w}(k+1)\right\|_{1} \\
\text { Subject to } \min _{\hat{W}(k+1)}\|\hat{w}(k+1)-\hat{w}(k)\|_{2}^{2} \leq \delta^{2}
\end{gathered}
$$

where $\delta^{2}$ is a applied constraint required to prevent the drastic change of weight update coefficients. $\delta^{2}$ should be as small as possible as it controls the convergence rate. By combining (3) \& (4) and applying the Lagrange multiplier, the cost function can be obtained as-

$$
J(\hat{w}(k+1))=\left\|e_{p}(k)\right\|_{1}+\beta\left[\|\hat{w}(k+1)-\hat{w}(k)\|_{2}^{2}-\delta^{2}\right]
$$

where $\beta$ is a Lagrange multiplier. By differentiating equation (5) w.r.t. weight vector $(\hat{w}(k+1))$ the equation $(5)$ can be written as- 


$$
\begin{aligned}
\frac{\partial J(\hat{w}(k+1))}{\partial(\hat{w}(k+1)} & =-\sum_{m=0}^{M-1} \operatorname{sgn}(\in(k-m)) x(k-m)+2 \beta[\hat{w}(k+1)-\hat{w}(k)] \\
& =X(k) \operatorname{sgn}\left(e_{p}(k)\right)+2 \beta[\hat{w}(k+1)-\hat{w}(k)]
\end{aligned}
$$

where the sign function is denoted as sgn (.).

$$
\operatorname{sgn}\left(\mathrm{e}_{\mathrm{p}}(\mathrm{k})\right)=[\operatorname{sgn} \varepsilon(\mathrm{k}), \operatorname{sgn} \varepsilon(\mathrm{k}-1), \ldots \ldots \ldots \ldots, \operatorname{sgn} \varepsilon(\mathrm{k}-\mathrm{L}+1)]^{\mathrm{T}}
$$

Equating the derivative with zero it is obtained that

$$
\hat{w}(k+1)=\hat{w}(k)+\frac{1}{2 \beta} X(k) \cdot \operatorname{sgn}\left(e_{p}(k)\right)
$$

Substituting (7) into the constraint (4), we obtained

$$
\frac{1}{2 \beta}=\frac{\delta}{\sqrt{\operatorname{sgn}\left(e_{p}^{T}(k)\right) X(k) X^{T}(k) \operatorname{sgn}\left(e_{p}(k)\right)}}
$$

Substituting (8) into (7), the weight update vector is obtained as

$$
\hat{w}(k+1)=\hat{w}(k)+\frac{\delta X(k) \cdot \operatorname{sgn}\left(e_{p}(k)\right)}{\sqrt{\operatorname{sgn}\left(e_{p}^{T}(k)\right) X(k) X^{T}(k) \operatorname{sgn}\left(e_{p}(k)\right)}}
$$

Since the a posteriori error vector $e_{p}(k)$ depends on $\hat{w}(k+1)$, it is reasonable to approximate posteriori error vector with a priori error vector $e(k)$ and the convergence rate is controlled by minimum disturbance $\delta$. The minimum value of $\delta$ ensures the convergence of algorithm. In conventional adaptive algorithm it serves the similar purpose as step size parameter. $\delta$ is replaced by step size parameter $\mu$ in the algorithm as the purpose of both are same.

By defining $X_{s}(k)=X(k) \operatorname{sgn}(e(k))$,

with $\operatorname{sgn}(e(k))=[\operatorname{sgn}(e(k)), \ldots \ldots \ldots \operatorname{sgn}(e(k-L+1))]^{T}$, the weight update equation for APSA is obtained as

$$
\hat{w}(k+1)=\hat{w}(k)+\mu \frac{X_{s}(k)}{\sqrt{X_{s}^{T}(k) X_{s}(k)+\epsilon}}
$$

where positive number $\in$ presents regularization parameter. The range of $\mu$ is $0<\mu \square 1$. Equation (10) shows that there is no need of matrix inversion and thus reduces the computational complexity.

\section{Experimental Setup and Results}

In this section, simulation results are discussed. The simulation performance is evaluated in reference to noise cancellation. This application is basically an interference cancellation problem, where the noise introduced in the original clean signal is being cancelled using adaptive filter. For simulation both, clean speech sentence "YAHA SAI LAGHBAG PANCH MEAL DAKSHIN PASCHIM MAI KATGHAR GAON HAI", and word "SHOONYA", is taken from Hindi Speech Database [15] and the various types of noises like "F16", "FACTORY", "CAR-NOISE" and "BABBLE16" which are taken from NOISEX-92 database [16] are used to corrupt above mentioned clean signals. Also the simulation is done at filter orders $5,10,15$ and 20 at $0 \mathrm{~dB},-5 \mathrm{~dB},-10 \mathrm{~dB},-5 \mathrm{~dB}$ and $20 \mathrm{~dB}$ input SNR. The performance of the said algorithms is measured in terms of SNR improvement with the decrement in MSE value. The performances of algorithms are also measured for convergence rate, robustness and the computational complexity. The SNR comparison of all the algorithms for various speech samples, both for word and sentence and for various noises, is shown in fig. 2 to fig. 6 . Also the convergence behavior of these 
algorithms is shown in fig. 7 to fig. 10. From fig. 2 to fig. 10 it is also observed that RAPSA algorithm performs best among the other two algorithms with SNR improvement at all filter order with faster convergence rate at low computational complexity. This is because of the fact that the matrix inversion operation is reduced in APSA algorithm.

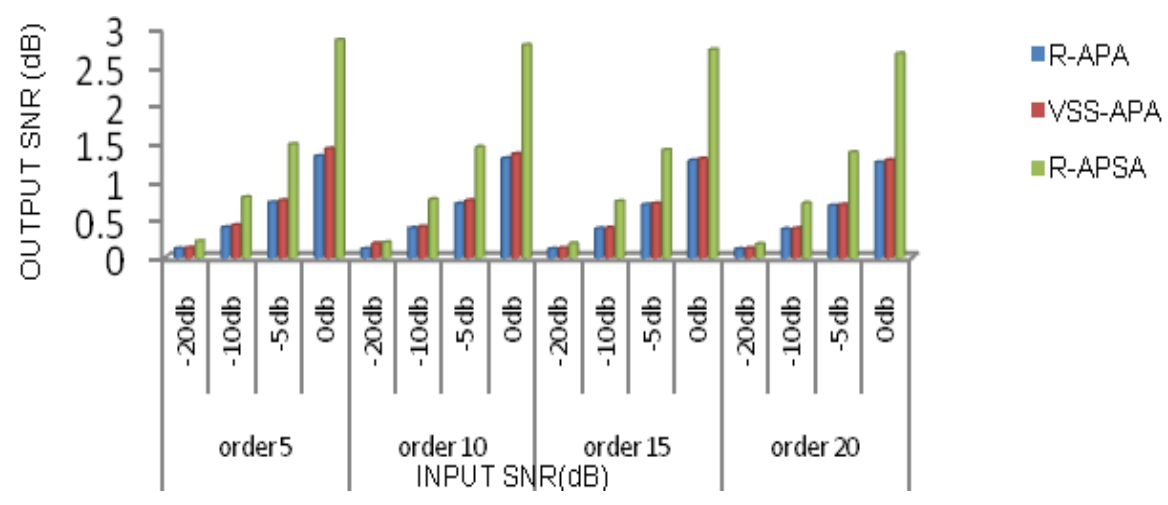

Figure 2. SNR Comparison of "Shoonya" Corrupted with "F16" Noise

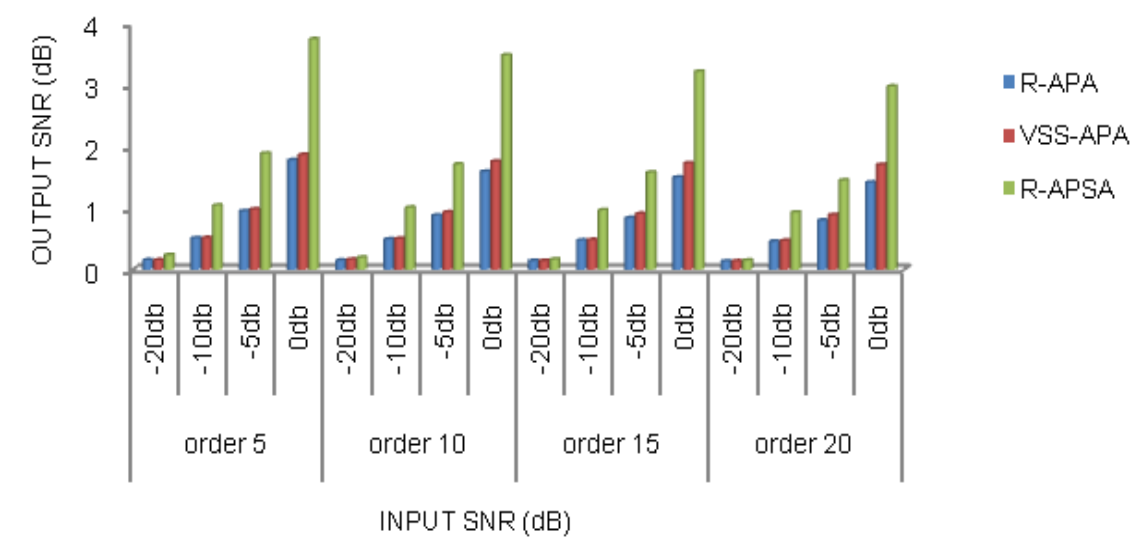

Figure 3. SNR Comparison of "Shoonya" Corrupted with "Factory" Noise

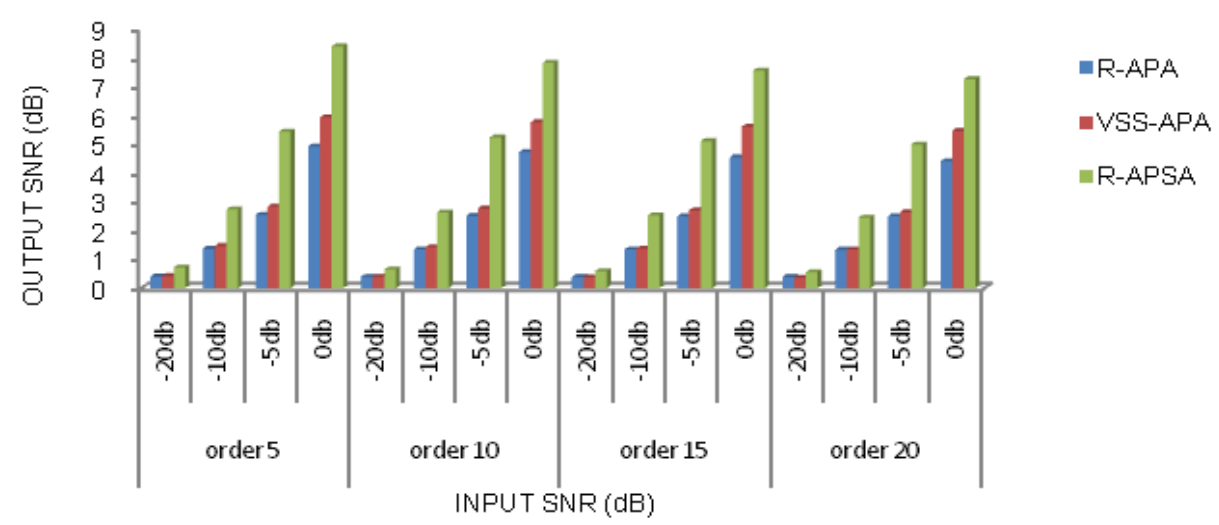

Figure 4. SNR Comparison of "Shoonya" Corrupted with "Bubble16" Noise 


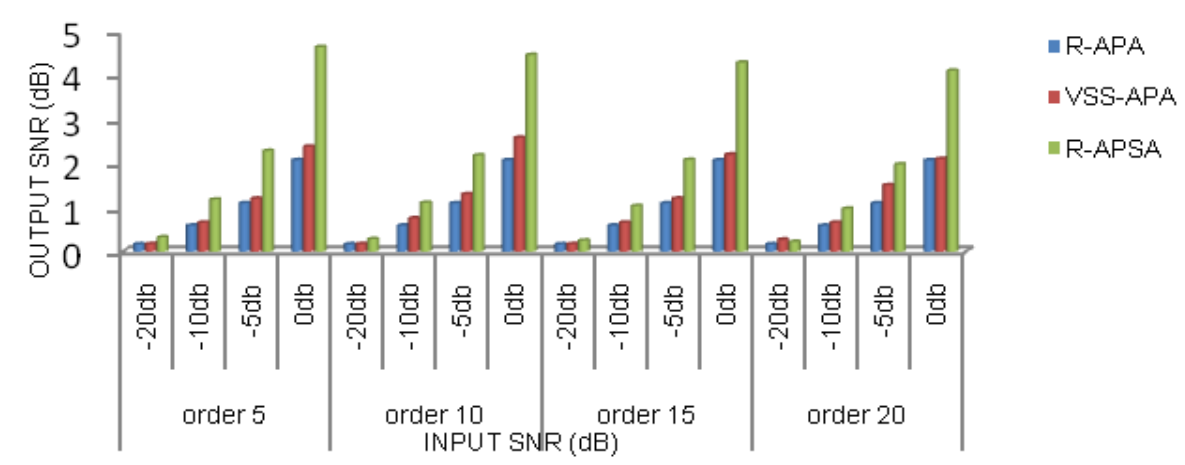

Figure 5. SNR Comparison of "Sentence" Corrupted with "Factory" Noise

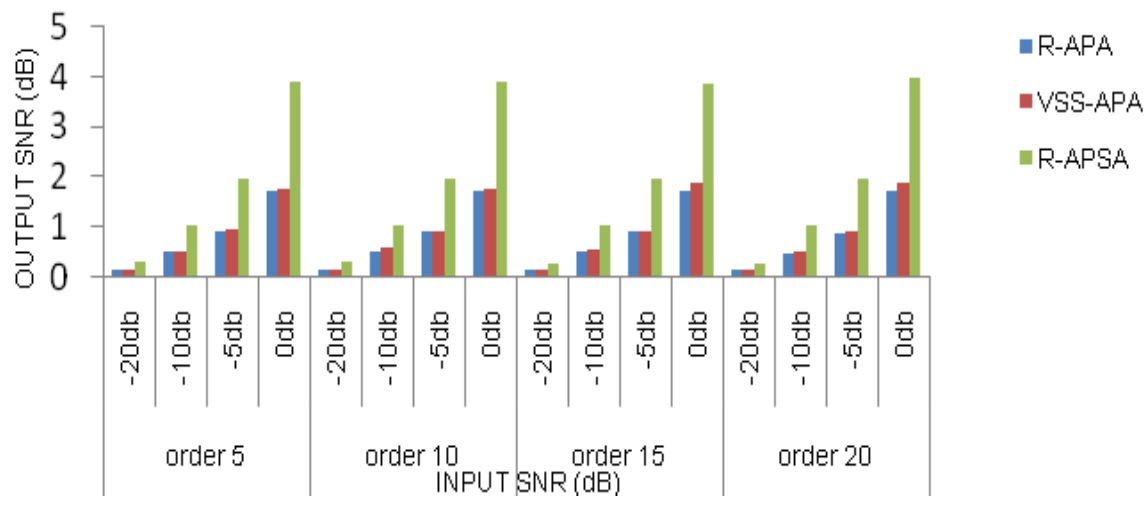

Figure 6. SNR Comparison of "Sentence" Corrupted with "F16" Noise

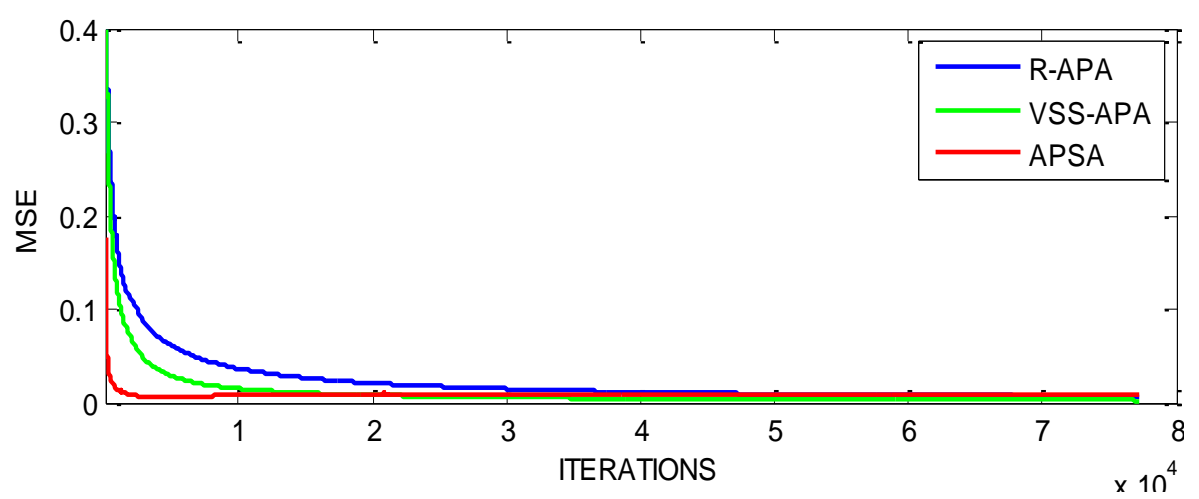

Figure 7. Convergence Behavior of "Sentence" Corrupted with "F16" Noise 


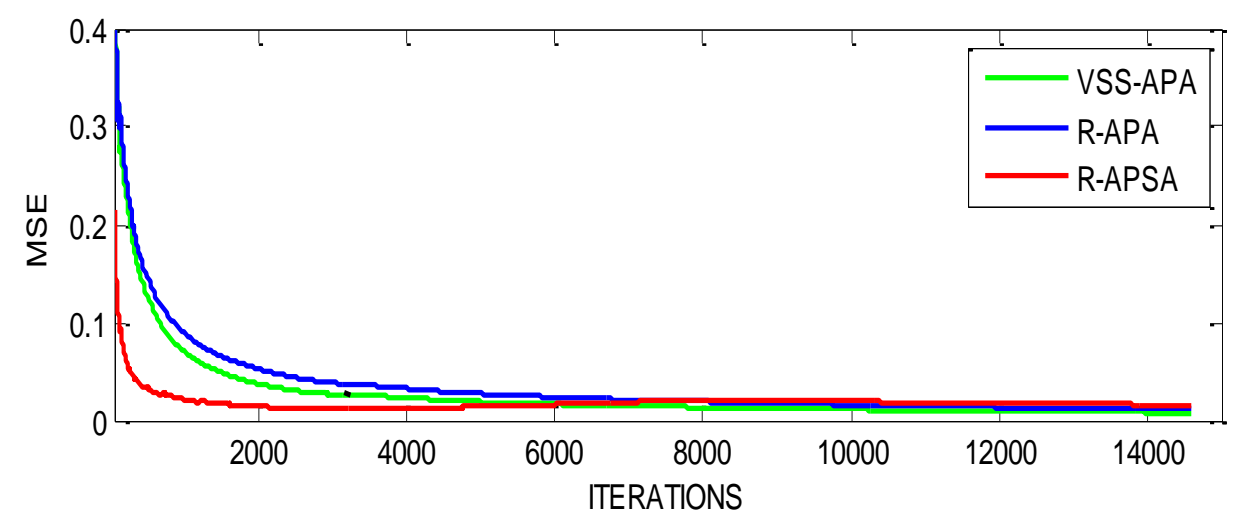

Figure 8. Convergence Behavior of "Shoonya" Corrupted with "F16" Noise

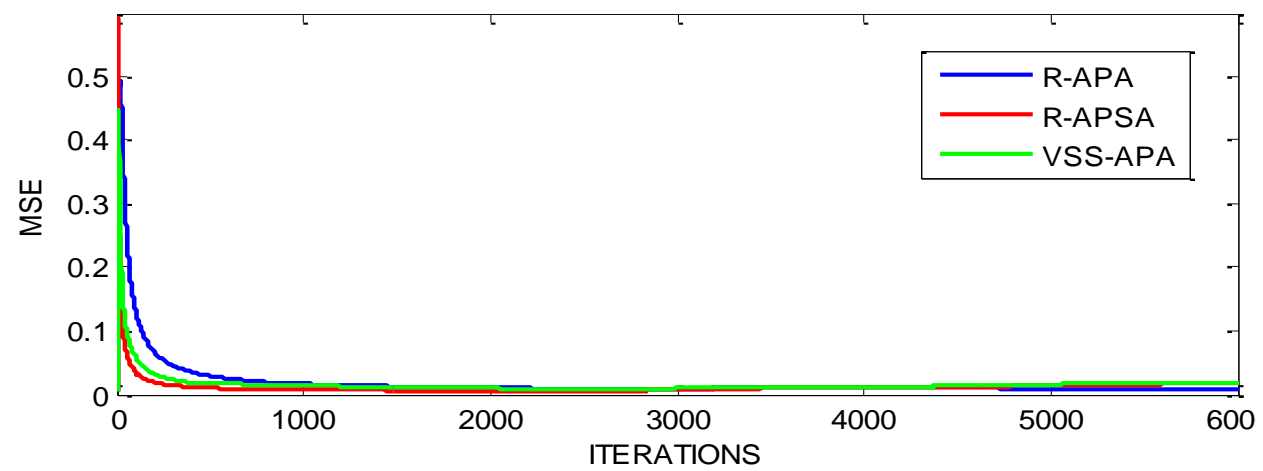

Figure 9. Convergence Behavior of "Shoonya" Corrupted with "Car" Noise

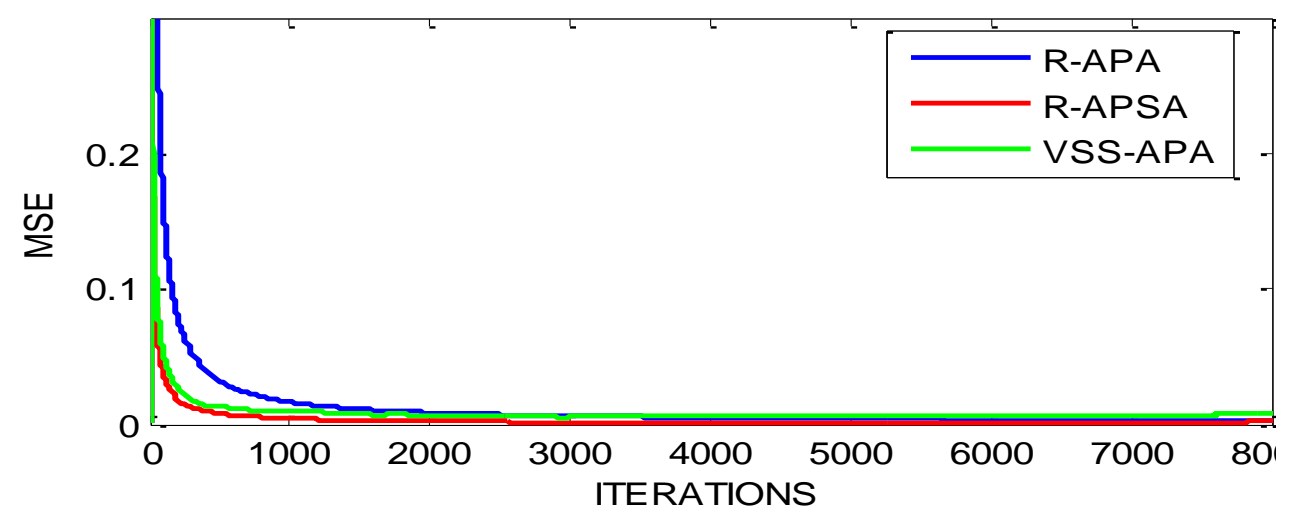

Figure 10. Convergence Behavior of "Sentence" Corrupted with "Car" Noise

The convergence behavior of R-APA, VSS-APA and R-APSA algorithm is evaluated by the normalized mean square error estimates. Table 1 shows the comparative analysis of MSE estimates for various clean signals corrupted with different noises. 
Table 1. Comparative Analysis of MSE Estimates

\begin{tabular}{|c|c|c|c|c|}
\hline $\begin{array}{l}\text { SAMPLE, } \\
\text { NOISE }\end{array}$ & $\begin{array}{c}\text { FILTER } \\
\text { ORDER, } \\
\text { INPUT } \\
\text { SNR } \\
\end{array}$ & R-APA & VSS-APA & R-APSA \\
\hline \multirow{2}{*}{$\begin{array}{l}\text { "SENTENCE", } \\
\text { "F16" }\end{array}$} & “5”, “-20" & 0.024578 & 0.023021 & 0.016594 \\
\hline & "5", "-10" & 0.010036 & 0.005156 & 0.002787 \\
\hline \multirow{2}{*}{$\begin{array}{l}\text { "SENTENCE" } \\
\text { "FACTORY" }\end{array}$} & "5", "-20" & 0.0218 & 0.0213 & 0.0066 \\
\hline & “5”, “-10" & 0.0105 & 0.0097 & 0.0016 \\
\hline \multirow{2}{*}{$\begin{array}{l}\text { "SHOONYA", } \\
\text { "FACTORY" }\end{array}$} & “5”, “-20” & 0.03066 & 0.03057 & 0.02276 \\
\hline & "5", "-10" & 0.005891 & 0.004439 & 0.004149 \\
\hline \multirow{2}{*}{$\begin{array}{l}\text { "SHOONYA", } \\
\text { "CAR NOISE" }\end{array}$} & "5", “-20" & 0.0199 & 0.01354 & 0.01152 \\
\hline & "5", "-10" & 0.0114618 & 0.007864 & 0.00695757 \\
\hline
\end{tabular}

The value of $\delta$ is taken in between 0.002 to 0.2 for the R-APA whereas it is 0.2 to 0.5 for VSS-APA. Also the value of $\mu$ is taken at 0.91 to 0.93 for the R-APSA. The performance of these algorithms are also examined with different projection orders and found that as the projection order increases the convergence behavior improved means algorithm achieves faster convergence with increased computational complexity. Therefore, by selecting a proper projection order the faster convergence rate can be achieved with lower computational complexity.

Table 2 shows the comparison of computational complexity of the said algorithms which are estimated per iterations. In this work projection order used is 2, filter order used is 5, 10, 15 and 20 and step size parameter varies between 0.002 to 0.9958. It is observed that step size variation affects the performance of the said algorithms. It is also observed that the smaller the value of step size reduces the convergence rate and the mean square error value and vice versa.

Table 2. Comparative Analysis of Computational Complexity

\begin{tabular}{|l|l|l|}
\hline \multicolumn{1}{|c|}{ Algorithm } & \multicolumn{1}{c|}{ Multiplications } & \multicolumn{1}{c|}{ Addition } \\
\hline R-APA & $\left(\mathrm{K}^{2}+2 \mathrm{~K}\right) \mathrm{L}+\mathrm{K}^{3}+\mathrm{K}^{2}$ & $\left(\mathrm{~K}^{2}+2 \mathrm{~K}\right) \mathrm{L}+\mathrm{K}^{3}$ \\
\hline VSS-APA & $\left(\mathrm{K}^{2}+6 \mathrm{~K}\right) \mathrm{L}+\mathrm{K}^{3}+\mathrm{K}^{2}+4 \mathrm{~K}+6$ & $\left(\mathrm{~K}^{2}+2 \mathrm{~K}\right) \mathrm{L}+\mathrm{K}^{3}+4 \mathrm{~K}+2$ \\
\hline R-APSA & $\mathrm{ML}+2 \mathrm{~L}+1$ & $2 \mathrm{ML}+\mathrm{L}$ \\
\hline
\end{tabular}

\section{Conclusions}

Many variants of affine projection algorithm have been proposed to achieve fast convergence rate, rapid tracking, improved output SNR ratio and low mean square error in the past two decades. This paper summarized several promising algorithms of APA family algorithms and presented a performance comparison by means of extensive simulations. In this paper R-APA, VSS-APA, and R-APSA algorithms are implemented for noise cancellation. These algorithms are robust as they performed well for both sentence and word corrupted with different types of noises, at the same time it shows better performance at lower input SNR levels. The R-APSA algorithm converges faster than that of R-APA and VSS-APA algorithm with highest SNR improvement with moderate mean square error value. The computational complexity of R-APSA algorithm achieved is smaller than other two algorithms. After extensive 
simulation it is observed that faster convergence rate is achieved when projection order increases.

\section{References}

[1] M. Kuo and D. R. Morgan, "Active Noise Control: a tutorial review", Proceedings of the IEEE, vol. 87, no. 6, (1999), pp. 943-973.

[2] M. Kuo, X. Kong and W. S. Gan, "Applications of Adaptive Feedback Active Noise Control System", IEEE Transactions on Control Systems Technology, vol. 11, no. 2, (2003), pp. 216220.

[3] B. F. Boroujeny, "Adaptive Filters, Theory and Applications", John Wiley and Sons, New York (1999).

[4] S. Haykin, "Adaptive Filter Theory”, 3rd ed. Prentice-Hall, Inc. Upper Saddle River, NJ, USA, (2002).

[5] A. H. Sayed, "Fundamentals of Adaptive Filtering", New York: Wiley, (2003).

[6] V. K. Gupta, M. Chandra and S. N. Saran, "Acoustic Echo and Noise Cancellation System for Hand-Free Telecommunication using Variable Step Size Algorithms”, Radio Engineering, vol. 22, no. 1, (2013), pp. 200-207.

[7] D. K Gupta, V. K. Gupta and M. Chandra, "A Review Paper on Linear and Nonlinear Acoustic Echo Cancellation, Proceedings of the $3^{\text {rd }}$ International Conference on Frontiers of Intelligent Computing: Theory and Applications, vol. 2, (2014), pp. 465-473.

[8] K. Ozeki and T. Umeda, "An Adaptive Filtering Algorithm using an Orthogonal Projection to an Affine Subspace and its Properties”, Electron. Commun. Jpn., vol. 67-A, no. 5, (1984), pp. 1927.

[9] Y. S. Choi, H. C. Shin and W. J. Song, "Affine Projection Algorithms with Adaptive Regularization Matrix", Icassp, (2006), pp. 201-204.

[10] H. C. Shin, A. H. Sayed and W. J. Song, "Variable Step-Size NLMS and Affine Projection Algorithms", IEEE Signal Proc. Letters, vol. 11, no. 2, (2004), pp. 132-135.

[11] H. Rey, L. Vega, S. Tressens and J. Benesty, "Variable Explicit Regularization in Affine Projection Algorithm: Robustness Issues and Optimal Choice," IEEE Trans. Signal Process., vol. 55, no. 5, (2007), pp. 2096-2108.

[12] C. Paleologu and J. Benesty, "A Variable Step-Size Affine Projection Algorithm Designed for Acoustic Echo Cancellation", IEEE Transactions on Audio, Speech, and Language Processing, vol. 16, no. 8, (2008), pp. 1446-1478.

[13] T. Shao, Rosa Yahong and J. Benesty, "An Affine Projection Sign Algorithm Robust Against Impulsive Interferences”, IEEE Signal Processing Letters, vol. 17, no. 4, (2010), pp. 327-330.

[14] Ni, Jingen and L. Feng, "Efficient Implementation of the Affine Projection Sign Algorithm", IEEE Signal Processing Letters, vol. 19, no. 1, (2012), pp. 24-26.

[15] K. Samudravijaya, "Hindi Speech Database", Proc. ICSLP00, Beijing, China, CDROM 00192.pdf.

[16] A. Varga, H. J. M. Steeneken and D. Jones, "The noisex-92 study on the effect of additive noise on automatic speech recognition system", Reports of NATO Research Study Group (RSG.10).

\section{Authors}

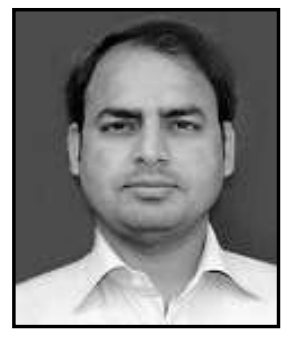

Deepak Kumar Gupta, he received the B.Sc degree in 1999 and M.Sc degree in 2001 from $\operatorname{Dr} \mathrm{R}$ M L Avadh University Faizabad,(U.P.), India. He has received M.Tech degree in Electronics Design and Technology from Centre of Electronics Design and Technology of India, Gorakhpur, U.P. in 2003. Currently he is pursuing Ph.D. from Dr A.P.J. A.K.T.U. Lucknow, India. His research interest includes signal and speech processing. $\mathrm{He}$ is working as an assistant professor at the Department of Electronics and Communication Engineering in Krishna Engineering College, Ghaziabad (UP), India. He has published more than 5 research papers in the area of speech and signal processing at national and international levels. He is a life member of IETE, member of IACSIT. 

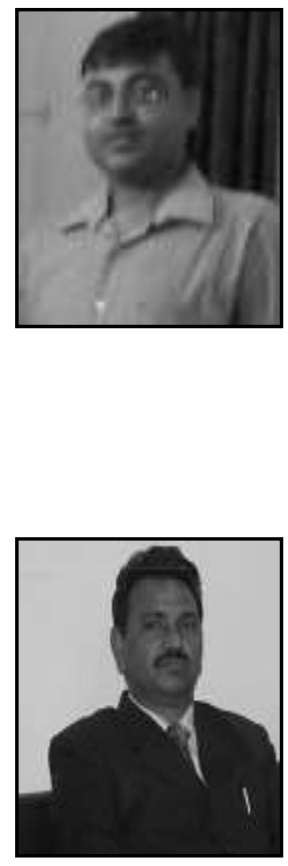

Vijay Kumar Gupta, he was born in 1976. He has received the B.Tech degree from MJP Rohilkhand University, Bareilly in 2001 and M.Tech degree in Instrumentation from DAVV, Indore in 2003. $\mathrm{He}$ has done Ph.D from Birla Institute of Technology, Mesra, Ranchi, India in 2013. His research interest includes signal and speech processing. He is working as professor and head at the Department of Electronics and Communication Engineering in Inderprestha Engineering College, Ghaziabad (UP), India. He has published more than 20 research papers in the area of speech and signal processing at national and international levels. He is a life member of ISTE, senior member of ICEIT and member of IEEE.

Mahesh Chandra, he was born in 1969. He received his B.Sc. from Agra University, Agra, India in 1990 and A.M.I.E. from I.E.I., Kolkatta, India in 1994. He received M.Tech. from J.N.T.U., Hyderabad-India in 2000 and Ph.D. from AMU, Aligarh, India in 2008. His areas of interest are speech, signal and image processing. He has worked as Reader and HOD in the Department of Electronics and Communication Engineering at S.R.M.S. College of Engineering and Technology, Bareilly, Indiafrom January 2000 to June 2005. Presently, he is working as professor in the Department of Electronics and Communication Engineering, B.I.T., Mesra, Ranchi (Jharkhand)India. He has published more than 166 research papers in the area of speech, signal and image processing at national and international levels. He is a Life Member of ISTE, Member of IEEE and IEI, Fellow member of IETE. 